2. To: (Receiving Organization)

Packaging Engineering

5. Proj./Prog./Dept./Div.:

PE/WMNW

8. Originator Remarks:

The attached SEP is being submitted for approval and release.

11. Receiver Renarks:

11A. Design Baseline Document?

[] Yes [X] no

3. Fron: (Originating Organization)
Packaging Engineering
6. Design Authority/ Design Agent/Cog.
Engr.:
W. A. McCormick

3. Fron: (Originating Organization) Design Authority/ Design Agent/Cog.

A. McCormick

4. Related EDT No.:
NA
7. Purchase order No.:
NA

9. Equip./Component No.:

NA

10. System/Bldg./Facility: $\mathrm{NA}$ NA

13. Permit/Permit Application No.: NA

14. Required Response Date: NA

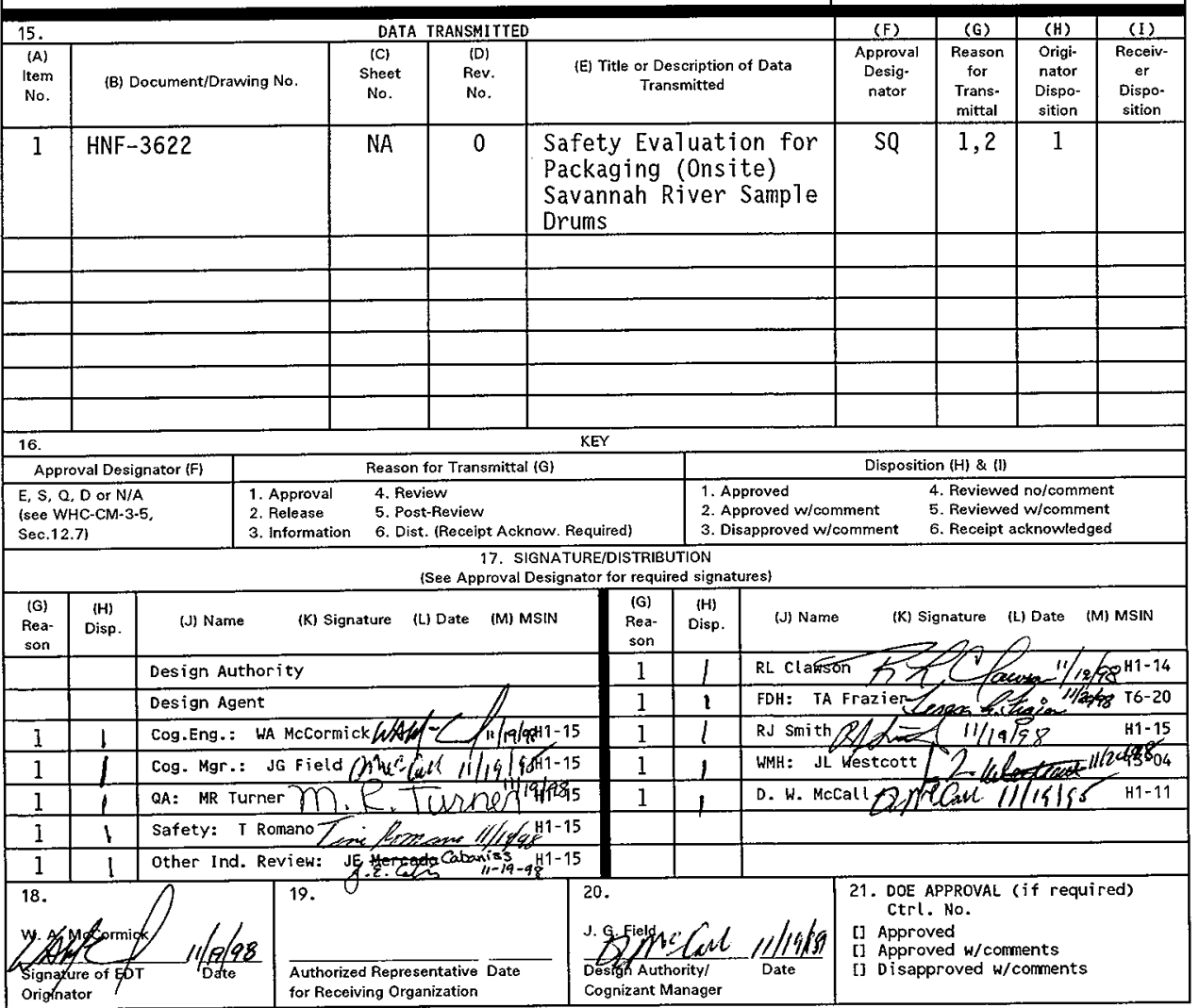

BD-7400-172-2 (05/96) GEF097 


\title{
Safety Evaluation for Packaging (Onsite) Savannah River Sample Drums
}

\author{
W. A. McCormick \\ Waste Management Federai Services, Inc., \\ Northwest Operations, Richland, WA 99352 \\ for Fluor Daniel Hanford, Inc. \\ U.S. Department of Energy Contract DE-AC06-96RL13200 \\ EDT/ECN: EDT 622971 \\ Org Code: R2162000 \\ CACN: 106519 \\ COA: EFOO \\ B\&R Code: \\ UC: 513 \\ Task Order: GS080003 \\ Project: 772031 \\ Crosswalk: 154 \\ Total Pages: 21
}

Key Words: $208 \mathrm{~L}$ drum, $30 \mathrm{~L}$ drum, 222-S Laboratory, T P1 ant

Abstract: This safety evaluation for packaging (SEP) evaluates and documents the safe onsite transport of $208 \mathrm{~L}$ (55-gal) and $30 \mathrm{~L}$ (8-gal) drums containing Savannah River Site liquid samples to facilities in the $200 \mathrm{~W}$ Area.

IRADEMARK DISCLAIMER. Reference herein to any specific commercial product, process, or service by trade nane, trademark, manufacturer, or otherwise, does not necessarily constitute or imply its endorsement, recommendation, or favoring by the United States Government or any agency thereof or its contractors or subcontractors.

Printed in the United States of America. To obtain copies of this document, contact: WHC/BCS Document Control Services, P.O. Box 1970, Mailstop H6-08, Richland WA 99352, Phone (509) 372-2420; Fax (509) 376-4989.
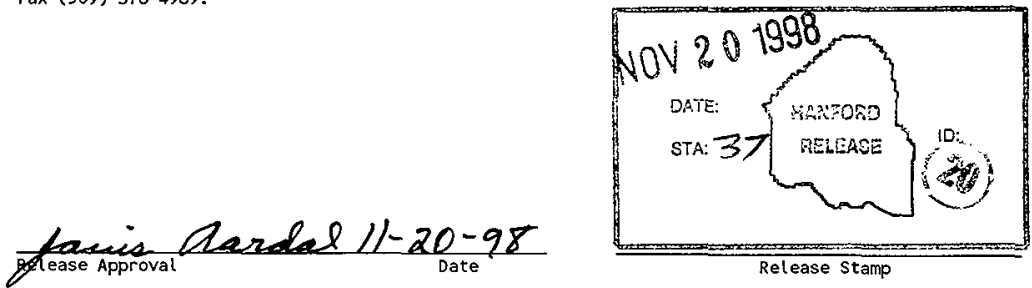

\section{Approved for Public Release}


HNF-3622, Rev. 0

\section{CONTENTS}

1.0 INTRODUCTION

1.1 GENERAL INFORMATION

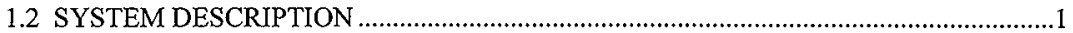

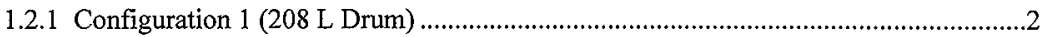

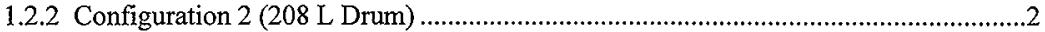

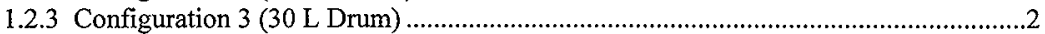

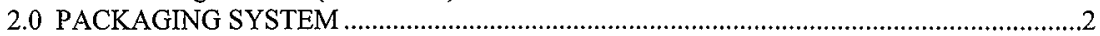

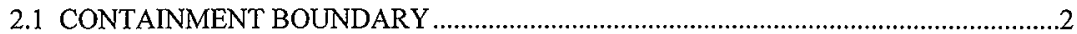

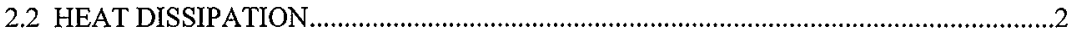

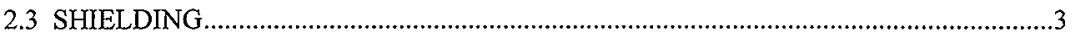

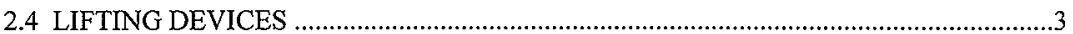

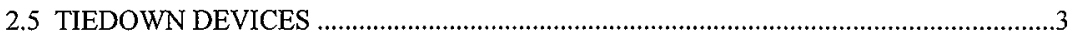

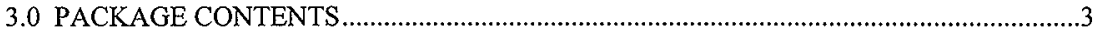

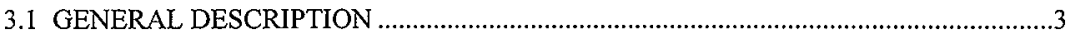

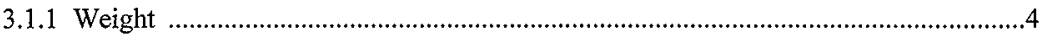

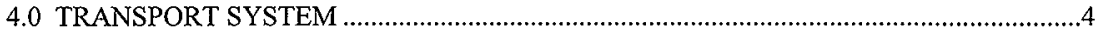

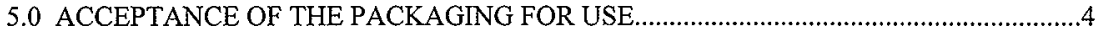

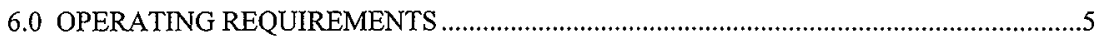

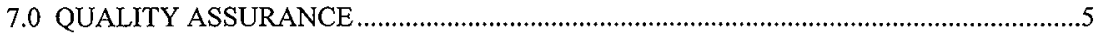

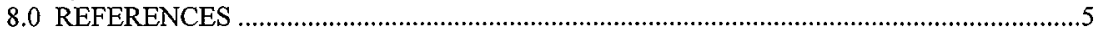

\section{APPENDICES}

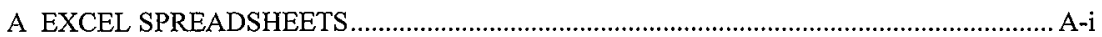

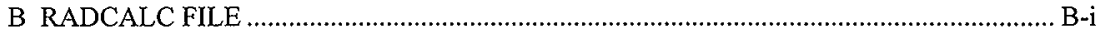

TABLE

Table 3-1. Package Identification and Configuration. 
HNF-3622, Rev. 0

\section{LIST OF TERMS}

$\begin{array}{ll}\text { ALARA } & \text { as low as reasonably achievable } \\ \mathrm{cm} & \text { centimeter } \\ \mathrm{gal} & \text { gallon } \\ \mathrm{in} . & \text { inch } \\ \mathrm{kg} & \text { kilogram } \\ \mathrm{L} & \text { liter } \\ \mathrm{lb} & \text { pound } \\ \mathrm{mrem} / \mathrm{h} & \text { millirem per hour } \\ \mathrm{mW} & \text { milliwatt }\end{array}$


HNF-3622, Rev. 0

\section{SAFETY EVALUATION FOR PACKAGING (ONSITE) \\ SAVANNAH RIVER SAMPLE DRUMS}

\subsection{INTRODUCTION}

\subsection{GENERAL INFORMATION}

The 222-S Laboratory is currently storing fourteen $208 \mathrm{~L}$ (55-gal) drums and three $30 \mathrm{~L}$ (8-gal) drums containing liquid samples in glass and/or polyethylene bottles. The original intent was to dispose of the sample material at the 222-S Laboratory; however, it has been determined that the chemical constituents of the material do not fall within the permit description for disposal at the laboratory. Consequently, the material requires disposal at the T Plant facility. As currently packaged, the amount of sample material contained in each drum may exceed the U.S. Department of Transportation limits for transport in the drums.

However, due to ALARA concerns, it is desired to transport the material to the $\Upsilon$ Plant or the Central Waste Complex as packaged rather than risk unnecessary worker exposure by repackaging the material. The radiological constituent source term for each of these drums falls well within the general limits prescribed in HNF-2209, Safety Analysis Report for Packaging (Onsite) Steel Drum (McCormick 1998). However, the method by which the sample bottles were packaged differs from the configuration described in McCormick (1998), and the presence of lead shielding in some of the drums is restricted by McCormick (1998). In addition, the packages are not vented; however, the total activity in each one is so low that this operation is unnecessary.

This safety evaluation for packaging authorizes the intra-area transport of these drums from the 222-S Laboratory to the T Plant or to the Central Waste Complex, as required.

\subsection{SYSTEM DESCRIPTION}

The basic component of each packaging consists of a U.S. Department of Transportation UN1A2 $208 \mathrm{~L}$ (55-gal) or $30 \mathrm{~L}$ (8-gal) steel drum. Two separate methods were used to package the sample bottles in the $208 \mathrm{~L}$ (55-gal) drums. There are also three $30 \mathrm{~L}$ (8-gal) UN1A2 drums authorized by this safety evaluation for packaging. All drums are restricted to a maximum radioactive constituent source term of less than or equal to $2 \mathrm{~A}_{2} \mathrm{~s}$, as defined by the 49 CFR 173, "Shippers - General Requirements for Shipments and Packagings." Specific drum contents descriptions are given in Appendix A. 


\subsubsection{Configuration 1 (208 L Drum)}

In this configuration, the sample bottles were placed in individual $30 \mathrm{~L}$ (8-gal) drums and separated using universal polyethylene material to provide dunnage and absorbency. Two $30 \mathrm{~L}$ (8-gal) drums were then placed in a single $208 \mathrm{~L}$ (55-gal) drum separated and supported by universal polypropylene material. In some cases, where dose rates necessitated, lead sheeting was added to the $30 \mathrm{~L}$ (8-gal) drums to attenuate gamma photon emissions prior to inserting them into the $208 \mathrm{~L}$ ( $55-\mathrm{gal}) \mathrm{drum}$. In these cases approximately $1.3 \mathrm{~cm} \mathrm{(1/2} \mathrm{in.)} \mathrm{of} \mathrm{lead} \mathrm{is} \mathrm{present}$ on the bottom of the drum and $0.95 \mathrm{~cm}(3 / 8 \mathrm{in}$.) on the sides.

\subsubsection{Configuration 2 (208 L Drum)}

In this configuration, an approximately $5.1 \mathrm{~cm}$ - (2-in.-) think layer of polymerpolyacrylate type material was placed in the bottom of the $208 \mathrm{~L}$ (55-gal) drum, and then a 90-mil liner was placed in the drum. Sample bottles were then placed individually in the drum, separated by universal polypropylene material. Each layer of bottles is also separated by universal polypropylene material.

\subsubsection{Configuration 3 (30 L Drum)}

The three $30 \mathrm{~L}$ (8-gal) drums are packaged similarly to configuration 1; however, only drum number 9602705 contains additional lead shielding.

\subsection{PACKAGING SYSTEM}

\subsection{CONTAINMENT BOUNDARY}

The containment boundary is provided by the $208 \mathrm{~L}$ (55-gal) or $30 \mathrm{~L}$ (8-gal) drum body, lid, and gasket.

\subsection{HEAT DISSIPATION}

Heat dissipation is by passive and conductive thermal radiation. All of the drums contain less than $3 \mathrm{~mW}$. Gas generation due to decay heat is not an issue. Venting is not required. 
HNF-3622, Rev. 0

\subsection{SHIELDING}

Shielding is provided by the contents and the packaging and in some cases by the addition of lead sheeting to attenuate gamma photon emissions. Dose rates on the external surfaces of the drums shall be limited to 1 rem or less.

\subsection{LIFTING DEVICES}

There are no lifting devices or attachments integral to the packagings. Lifting devices and attachments used to lift individual $208 \mathrm{~L}$ (55-gal) drums shall have load ratings in excess of $670 \mathrm{~kg}(1450 \mathrm{lb})$ and load ratings in excess of $102 \mathrm{~kg}(225 \mathrm{lb})$ for the $30 \mathrm{~L}$ (8-gal) drums.

\subsection{TIEDOWN DEVICES}

There are no tiedown devices that are a structural part of the packaging systems. Drums shall be secured to the transport vehicle as specified in Part A, Section 4.2, of McCormick (1998).

\subsection{PACKAGE CONTENTS}

\subsection{GENERAL DESCRIPTION}

The chemical and radioactive material present in the liquid sample bottles is primarily derived from analytical activities performed at the Savannah River Site on Hanford tank farm material. Radionuclides listed on analytical result data sheets are ${ }^{60} \mathrm{Co},{ }^{90} \mathrm{Sr},{ }^{90} \mathrm{Y},{ }^{99} \mathrm{Tc},{ }^{137} \mathrm{Cs}$, ${ }^{154} \mathrm{Eu},{ }^{237} \mathrm{~Np},{ }^{238} \mathrm{Pu},{ }^{239} \mathrm{Pu},{ }^{241} \mathrm{Pu}$, and ${ }^{241} \mathrm{Am}$. All of the drums contain fissile excepted (49 CFR 173) quantities of fissile material. The $\mathrm{pH}$ of the material ranges from 1 to greater than 12.5; however, bases are separated from acids. Each drum contains material, packaged in glass or poly bottles, that is either in the low-to-neutral $\mathrm{pH}$ range or in the high $\mathrm{pH}$ range. No mixing of high and low $\mathrm{pH}$ material is present. The sample bottles range in size from $125 \mathrm{ml}$ to $1 \mathrm{~L}$. The largest amount of liquid material present in any single drum less than $40 \mathrm{~L}$.

All but three of the drums contain less than a Type A quantity of radioactive material. The three drums that exceed Type A quantities do so by a very small amount. None of these drums contain more than two $\mathrm{A}_{2} \mathrm{~S}$, which is considerably less than the McCormick (1998) limit of 2,500 $\mathrm{A}_{2}$ s. Table 3-1 shows a general description of the quantity of radioactive material and configuration for each package. 
HNF-3622, Rev. 0

Table 3-1. Package Identification and Configuration.

\begin{tabular}{|l|c|c|c|c|}
\hline $\begin{array}{c}\text { Drum I.D. } \\
\text { number }\end{array}$ & $\begin{array}{c}\text { External dose } \\
\text { rate }(\mathrm{mrem} / \mathrm{h})\end{array}$ & $\begin{array}{c}\text { Total number } \\
\text { of } \mathrm{A}_{2} \mathrm{~S}\end{array}$ & $\mathrm{pH}$ & $\begin{array}{c}\text { Shielding } \\
\text { present? (Y/N) }\end{array}$ \\
\hline 9601758 & $>100$ & 0.0890 & $2-6$ & $\mathrm{Y}$ \\
\hline 9601835 & 70 & 0.1200 & Caustic & $\mathrm{Y}$ \\
\hline $9607625^{*}$ & $>100$ & 0.6000 & $>12.5$ & $\mathrm{Y}$ \\
\hline 9607979 & 1.3 & 0.0008 & Organic & $\mathrm{N}$ \\
\hline 9607983 & $<0.5$ & 0.0006 & $1-7$ & $\mathrm{~N}$ \\
\hline 9607984 & 4 & 0.0018 & $1-6$ & $\mathrm{~N}$ \\
\hline 9608010 & $<0.5$ & $2.56 \mathrm{E}-4$ & Organic & $\mathrm{N}$ \\
\hline 9608011 & $<0.5$ & 0.0013 & $1-7$ & $\mathrm{~N}$ \\
\hline 9608059 & 5 & 0.0004 & $1-7$ & $\mathrm{~N}$ \\
\hline 9608064 & 0.5 & 0.1700 & $5->12.5$ & $\mathrm{~N}$ \\
\hline 9608111 & $>100$ & 1.2700 & $>12.5$ & $\mathrm{Y}$ \\
\hline 9701835 & $>100$ & 1.0200 & Caustic & $\mathrm{Y}$ \\
\hline 9701844 & $>100$ & 1.1900 & $1-2$ & $\mathrm{Y}$ \\
\hline 9703473 & 100 & 0.0609 & $1-2$ & $\mathrm{~N}$ \\
\hline 9703517 & 100 & 0.0096 & $11-12.5$ & $\mathrm{~N}$ \\
\hline $9802764^{*}$ & $<0.5$ & $6.83 \mathrm{E}-4$ & Organic & $\mathrm{N}$ \\
\hline $9802658^{*}$ & $<0.5$ & 0.0020 & $1-2$ & $\mathrm{~N}$ \\
\hline
\end{tabular}

*30 L (8-gal) drum

\subsubsection{Weight}

The $208 \mathrm{~L}$ (55-gal) drums are limited to a maximum gross weight of $454 \mathrm{~kg}(1,000 \mathrm{lb})$. The $30 \mathrm{~L}$ (8-gal) drums are limited to a maximum gross weight of $96 \mathrm{~kg}(211 \mathrm{lb})$.

\subsection{TRANSPORT SYSTEM}

The transport system shall meet the requirements of Part A, Section 4.0 , of McCormick (1998).

\subsection{ACCEPTANCE OF THE PACKAGING FOR USE}

The packaged drums shall meet the requirements of Part A, Section 5.2, of McCormick (1998) except for the exceptions stated in this safety evaluation for packaging. 
HNF-3622, Rev. 0

\subsection{OPERATING REQUIREMENTS}

The operating requirements of Part A, Section 6.1, of McCormick (1998) shall be followed with the exception of item 4 of that section. Venting is not required for this operation.

\subsection{QUALITY ASSURANCE}

The quality assurance requirements of Part A, Section 7.0, of McCormick (1998) shall be met.

\subsection{REFERENCES}

49 CFR 173, "Shippers - General Requirements for Shipments and Packagings," Code of Federal Regulations, as amended.

McCormick, W. A., Safety Analysis Report for Packaging (Onsite) Steel Drum, HNF-2209, Rev. 0, prepared by Waste Management Federal Services, Inc., Northwest Operations for Fluor Daniel Hanford, Inc., Richland, Washington. 
HNF-3622, Rev. 0

APPENDIX A

EXCEL SPREADSHEETS 


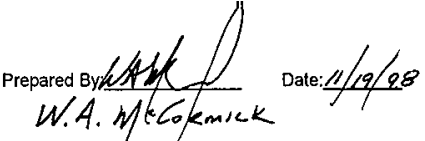

Checked By:G.8.G Date: $1 / 19 \cdot 98$

Savannah River Sample Drums

All Radionuclide Activities are listed in mCi (millicuries)

\section{Drum $\$ 9608059$}

\begin{tabular}{|c|c|c|c|c|c|c|c|c|c|c|c|c|}
\hline & & & Sr90 & Y90 & Tc99 & Cs137 & Eu154 & Np237 & Pu238 & Pu239 & Pu241 & Am241 \\
\hline$A 2$ value (Ci) & & 10.8 & 2.7 & $1.00 E+10$ & 24.3 & 13.5 & 13.5 & 5.41E-03 & 5.41E-03 & 5.41E-03 & $\quad 0.27$ & 5.41E-03 \\
\hline$\#$ of $A 2 \mathrm{~s}$ & $3.57 \mathrm{E}-04$ & $1.28 \mathrm{E}-06$ & $5.26 \mathrm{E}-05$ & $3.94 \mathrm{E}-15$ & 8.78E-07 & $6.04 E-05$ & $5.42 \mathrm{E}-06$ & 1.94E-07 & 5.01E-07 & $3.20 \mathrm{E}-05$ & $5.65 \mathrm{E}-07$ & 2.03E-04 \\
\hline
\end{tabular}

Bottle\#
D-2-2
D-3-2
D-4-2
D5-2
B-1-2
B-2-2
B-3-2
B-4-2
B-5-2
B-6-2
B-7
B-8
B-9
B-10
B-11-2
B-12
B-13
B-14
B-15
B-16
B-17-2
B-18-2
B-19-2

$\begin{array}{rrr}0.0037 & 0.038 & 0.0038 \\ 0.0037 & 0.038 & 0.0038 \\ 0.0037 & 0.038 & 0.0038 \\ 0.0027 & 0.0278 & 0.0278 \\ & 3.70 \mathrm{E}-06 & 3.70 \mathrm{E}-06 \\ & 3.70 \mathrm{E}-06 & 3.70 \mathrm{E}-06 \\ & 7.80 \mathrm{E}-06 & 7.80 \mathrm{E}-06 \\ & 3.70 \mathrm{E}-06 & 3.70 \mathrm{E}-06 \\ & 7.80 \mathrm{E}-06 & 7.80 \mathrm{E}-06 \\ & 1.20 \mathrm{E}-05 & 1.20 \mathrm{E}-05 \\ & 7.80 \mathrm{E}-06 & 7.80 \mathrm{E}-06 \\ & 7.80 \mathrm{E}-06 & 7.80 \mathrm{E}-06 \\ & 7.80 \mathrm{E}-06 & 7.80 \mathrm{E}-06 \\ & 5.70 \mathrm{E}-06 & 5.70 \mathrm{E}-06 \\ & 7.80 \mathrm{E}-06 & 7.80 \mathrm{E}-06 \\ & 1.20 \mathrm{E}-05 & 1.20 \mathrm{E}-05 \\ & 7.80 \mathrm{E}-06 & 7.80 \mathrm{E}-06 \\ & 7.80 \mathrm{E}-06 & 7.80 \mathrm{E}-06 \\ & 1.20 \mathrm{E}-05 & 1.20 \mathrm{E}-05 \\ & 1.20 \mathrm{E}-05 & 1.20 \mathrm{E}-05 \\ & 7.80 \mathrm{E}-06 & 7.80 \mathrm{E}-06 \\ & 1.20 \mathrm{E}-05 & 1.20 \mathrm{E}-05 \\ & 7.80 \mathrm{E}-06 & 7.80 \mathrm{E}-06\end{array}$

$\begin{array}{rr}0.0055 & 0.0368 \\ 0.0055 & 0.0368 \\ 0.0055 & 0.0368 \\ 0.004 & 0.0269 \\ 3.00 \mathrm{E}-05 & 0.0269 \\ 3.00 \mathrm{E}-05 & 0.0269 \\ 4.00 \mathrm{E}-05 & 0.0368 \\ 3.00 \mathrm{E}-05 & 0.0269 \\ 4.00 \mathrm{E}-05 & 0.0368 \\ 6.20 \mathrm{E}-05 & 0.0364 \\ 4.00 \mathrm{E}-05 & 0.0368 \\ 4.00 \mathrm{E}-05 & 0.0368 \\ 4.00 \mathrm{E}-05 & 0.0368 \\ 3.00 \mathrm{E}-05 & 0.0269 \\ 4.00 \mathrm{E}-05 & 0.0368 \\ 6.20 \mathrm{E}-05 & 0.0364 \\ 4.00 \mathrm{E}-05 & 0.0368 \\ 4.00 \mathrm{E}-05 & 0.0368 \\ 6.20 \mathrm{E}-05 & 0.0364 \\ 6.20 \mathrm{E}-05 & 0.0364 \\ 4.00 \mathrm{E}-05 & 0.0368 \\ 6.20 \mathrm{E}-05 & 0.0564 \\ 4.00 \mathrm{E}-05 & 0.0368\end{array}$

$\begin{array}{llllll}0.0196 & 2.80 \mathrm{E}-07 & 6.52 \mathrm{E}-07 & 5.63 \mathrm{E}-05 & 4.09 \mathrm{E}-05\end{array}$

0.0003

$0.0196 \quad 280 \mathrm{E}-07 \quad 6.52 \mathrm{E}-07 \quad 5.63 \mathrm{E}-05 \quad 4.09 \mathrm{E}-05 \quad 0.0003$

$\begin{array}{lllllll}0.0196 & 2.80 \mathrm{E}-07 & 6.53 \mathrm{E}-07 & 5.63 \mathrm{E}-05 & 4.09 \mathrm{E}-05 & 0.0003 \\ 0.0144 & 2.10 \mathrm{E}-07 & 4.78 \mathrm{E}-07 & 4.13 \mathrm{E}-06 & 3.00 \mathrm{E}-05 & 0.0002\end{array}$
4.78E-07 4.13E-06 3.00E-05
3.30E-08

$9.75 \mathrm{E}-09 \quad 9.75 \mathrm{E}-09$

$1.33 \mathrm{E}-08 \quad 1.33 \mathrm{E}-08$

3.30E-08

4.40E-08

$9.75 \mathrm{E}-09 \quad 9.75 \mathrm{E}-09$

$1.33 \mathrm{E}-08 \quad 1.33 \mathrm{E}-08 \quad 4.40 \mathrm{E}-08$

2.04E-08 2.04E-08

$4.40 \mathrm{E}-08$
$6.80 \mathrm{E}-08$

$1.33 \mathrm{E}-08 \quad 1.33 \mathrm{E}-08$

4.40E-08

$1.33 \mathrm{E}-08 \quad 1.33 \mathrm{E}-08 \quad 4.40 \mathrm{E}-08$

$1.33 \mathrm{E}-08 \quad 1.33 \mathrm{E}-08$

9.75E-09 $9.75 \mathrm{E}-09$

4.40E-08

3.30E-08

$\begin{array}{lll}1.33 \mathrm{E}-08 & 1.33 \mathrm{E}-08 & 4.40 \mathrm{E}-08 \\ 2.04 \mathrm{E}-08 & 2.04 \mathrm{E}-08 & 6.80 \mathrm{E}-08\end{array}$

$\begin{array}{lll}2.04 \mathrm{E}-08 & 2.04 \mathrm{E}-08 & 6.80 \mathrm{E}-08 \\ 1.33 \mathrm{E}-08 & 1.33 \mathrm{E}-08 & 4.40 \mathrm{E}-08\end{array}$

$1.33 E-08 \quad 1.33 E-08 \quad 4.40 E-08$

2.04E-08 2.04E-08

$\begin{array}{lll}2.04 \mathrm{E}-08 & 2.04 \mathrm{E}-08 & 6.80 \mathrm{E}-08\end{array}$

$\begin{array}{lll}1.33 E-08 & 1.33 E-08 & 4.40 E-08\end{array}$

2.04E-08 2.04E-08 $6.80 \mathrm{E}-08$

$\begin{array}{lll}1.33 E-08 & 1.33 E-08 & 4.40 E-08\end{array}$

Total

0.107798

0.107798

0.107798

0.103835

0.026937

0.026937

0.036856

0.026937

0,036856

0.036486

0.036856

0.036856

0.036856

0.026941

0.036856

0.036486

0.036856

0.036856

0.036486

0.036486

0.036856

0.056486

0.036856

$0.1419548 \quad 0.039355$

0.02133

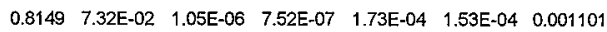

1.10597 


\section{Drum \#9608064}

\begin{tabular}{|c|c|c|c|c|c|c|c|c|c|c|c|c|}
\hline & & & Sr90 & Y90 & Tc99 & Cs137 & Eu154 & Np237 & Pu238 & Pu239 & Pu241 & Am241 \\
\hline Val & & $\begin{array}{r}10.8 \\
\end{array}$ & 2.7 & $7 \quad 1.00 E+10$ & 24.3 & $\begin{array}{r}13.5 \\
83.5\end{array}$ & $\begin{array}{r}13.5 \\
75 F\end{array}$ & 6.41E-03 & 6.41E-03 & 5.41E-03 & 0.27 & 5.41E-03 \\
\hline & 1.67E-01 & 1.67E-07 & $6.93 \mathrm{E}-03$ & $13 \quad 1.87 \mathrm{E}-12$ & $1.16 E-04$ & $8.06 \mathrm{E}-03$ & 7.15E-05 & $2.64 \mathrm{E}-05$ & $2.51 \mathrm{E}-04$ & $1.04 \mathrm{E}-01$ & $1.54 E-04$ & $4.80 \mathrm{E}-02$ \\
\hline
\end{tabular}

Bottle\#

BNFL-Vit-156

BNFL-Vit-155

BNFL-B-Filtrate-6

BNFL-B-4

BNFL-Vit-163

D-1-2 Organic ???

离

Total

\begin{tabular}{|c|c|c|c|c|}
\hline \multirow{3}{*}{$\begin{array}{l}\text { A2 value (Ci) } \\
\text { \# of A2s }\end{array}$} & \multicolumn{2}{|c|}{ Co60 } & 990 & Y90 \\
\hline & & 10.8 & 2.7 & $1.00 \mathrm{E}+$ \\
\hline & $1.75 \mathrm{E}-03$ & 8.13E-06 & $3.37 \mathrm{E}-0.04$ & $9.09 \mathrm{E}$ \\
\hline \multicolumn{5}{|l|}{ Bottle\# } \\
\hline D-1 & & 0.0027 & 0.0278 & \\
\hline D-2 & & 0.0027 & 0.0278 & 0.02 \\
\hline D-3 & & 0.0056 & 0.0583 & 0.05 \\
\hline D-4 & & 0.0056 & 0.0583 & 0.0583 \\
\hline D- 6 & & 0.0027 & 0.0278 & 0.02 \\
\hline D-7 & & 0.0027 & 0.0278 & 0.0278 \\
\hline D-8 & & 0.0037 & 0.038 & 0.0 \\
\hline D-9 & & 0.0027 & 0.0278 & 0.02 \\
\hline D-11 & & 0.0027 & 0.0278 & 0.02 \\
\hline D-12 & & 0.0027 & 0.0278 & 0.02 \\
\hline D-13 & & 0.0056 & 0.0583 & 0.05 \\
\hline D-14 & & 0.0056 & 0.0583 & \\
\hline D-15 & & 0.0037 & 0.038 & \\
\hline D-16 & & 0.0037 & 0.038 & \\
\hline
\end{tabular}

$\begin{array}{rr}0.4036 & 0.4036 \\ & \\ 0.0167 & 0.0167 \\ 0.0025 & 0.0025 \\ 18.2966 & 18.2966 \\ & \\ & \\ 18.7194 & 18.7194\end{array}$

\subsection{6}

0.0003

0.0862

0.0129

2.6567

0.3906

78.6571

11.8131

0.0018

0.0018

$18.7194 \quad 18.7194$

$2.8147 \quad 108.8053$ $\begin{array}{lllll}0.0208 & 3.00 E-06 & 2.86 E-05 & 1.24 E-04 & 8.98 E-04\end{array}$

8.45E-08 $8.45 \mathrm{E}-08$

$285 \mathrm{E}-05 \quad 2.85 \mathrm{E}-05$

$4.28 \mathrm{E}-06 \quad 4.28 \mathrm{E}-06$

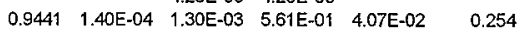

0.0056

0.0001

0.2597
24.3

$\begin{array}{llllll}\text { Cs137 Eu154 Np237 Pu238 Pu239 Pu241 Am241 } & \text { Am }\end{array}$ $\begin{array}{llllllll}5.47 \mathrm{E}-06 & 1.33 \mathrm{E}-04 & 3.48 \mathrm{E}-05 & 1.25 \mathrm{E}-06 & 2.95 \mathrm{E}-06 & 2.50 \mathrm{E}-05 & 3.62 \mathrm{E}-06 & 1.20 \mathrm{E}-03\end{array}$
Total Total
1.283853
0.2337
78.77686
11.83101
58.76363

150.889
Total

0.103835 0.103835

0.217673

0.217673

0.103835

0.103835

0.141947

0.103835

0.103835

0.103835

0.217673

0.217673

0.141947

0.141947 


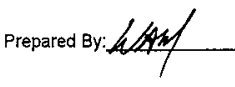

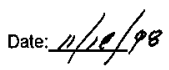

$\begin{array}{lrr}0.0056 & 0.0583 & 0.0583 \\ 0.0056 & 0.0583 & 0.0583 \\ 0.0056 & 0.0583 & 0.0583 \\ 0.0056 & 0.0583 & 0.0583 \\ 0.0037 & 0.038 & 0.038 \\ 0.0037 & 0.038 & 0.038 \\ 0.0056 & 0.0583 & 0.0583 \\ & 1.20 \mathrm{E}-05 & 1.20 \mathrm{E}-05 \\ & 1.20 \mathrm{E}-05 & 1.20 \mathrm{E}-05 \\ & 1.20 \mathrm{E}-05 & 1.20 \mathrm{E}-05 \\ & 1.20 \mathrm{E}-05 & 1.20 \mathrm{E}-05 \\ & 1.20 \mathrm{E}-05 & 1.20 \mathrm{E}-05 \\ & 1.20 \mathrm{E}-05 & 1.20 \mathrm{E}-05 \\ & 1.20 \mathrm{E}-05 & 1.20 \mathrm{E}-05 \\ & 1.20 \mathrm{E}-05 & 1.20 \mathrm{E}-05 \\ & 7.80 \mathrm{E}-06 & 7.80 \mathrm{E}-06 \\ & 1.20 \mathrm{E}-05 & 1.20 \mathrm{E}-05 \\ & 1.20 \mathrm{E}-05 & 1.20 \mathrm{E}-05 \\ & 1.20 \mathrm{E}-05 & 1.20 \mathrm{E}-05 \\ & 1.20 \mathrm{E}-05 & 1.20 \mathrm{E}-05 \\ 1.20 \mathrm{E}-05 & 1.20 \mathrm{E}-05 \\ & 1.20 \mathrm{E}-05 & 1.20 \mathrm{E}-05 \\ & 1.20 \mathrm{E}-05 & 1.20 \mathrm{E}-05 \\ & 5.70 \mathrm{E}-06 & 5.70 \mathrm{E}-06\end{array}$

Total
Checked By: \& EGG- Date: $11 \cdot 19.48$

$\begin{array}{llllllll}0.0085 & 0.0564 & 3.01 E-02 & 4.30 E-07 & 1.00 E-06 & 8.64 E-06 & 6.27 E-05\end{array}$ $\begin{array}{lllllll}0.0085 & 0.0564 & 3.01 E-02 & 4.30 \mathrm{E}-07 & 1.00 \mathrm{E}-06 & 8.64 \mathrm{E}-06 & 6.27 \mathrm{E}-05\end{array}$

$\begin{array}{llllllll}0.0085 & 0.0564 & 3.01 E-02 & 4.30 \mathrm{E}-07 & 1.00 \mathrm{E}-06 & 8.64 \mathrm{E}-06 & 6.27 \mathrm{E}-05\end{array}$

$\begin{array}{llllllll}0.0085 & 0.0564 & 3.01 \mathrm{E}-02 & 4.30 \mathrm{E}-07 & 1.00 \mathrm{E}-06 & 8.64 \mathrm{E}-06 & 6.27 \mathrm{E}-05\end{array}$

$\begin{array}{llllllll}0.0055 & 0.0368 & 0.0196 & 2.80 \mathrm{E}-07 & 6.52 \mathrm{E}-07 & 5.63 \mathrm{E}-06 & 4.09 \mathrm{E}-05\end{array}$

$\begin{array}{llllllll}0.0055 & 0.0368 & 0.0196 & 2.80 \mathrm{E}-07 & 6.52 \mathrm{E}-07 & 5.63 \mathrm{E}-06 & 4.09 \mathrm{E}-05\end{array}$

0.0085

$\begin{array}{llllll}0.0564 & 3.01 E-02 & 4.30 E-07 & 1.00 E-06 & 8.64 E-06 & 6.27 E-05\end{array}$

0.0564

2.04E-08 2.04E-08

2.04E-08 2.04E-08

2.04E-08 2.04E-08

2.04E-08 2.04E-08

2.04E-08 2.04E-08

2.04E-08 2.04E-08

2.04E-08 2.04E-08

2.04E-08 2.04E-08

$1.33 \mathrm{E}-08 \quad 1.33 \mathrm{E}-08$

2.04E-08 2.04E-08

$2.04 \mathrm{E}-08 \quad 2.04 \mathrm{E}-08$

2.04E-08 2.04E-08

2.04E-08 2.04E-08

$2.04 \mathrm{E}-08 \quad 2.04 \mathrm{E}-08$

2.04E-08 2.04E-08

2.04E-08 2.04E-08

$9.75 \mathrm{E}-09 \quad 9.75 \mathrm{E}-09$

$\begin{array}{ll}6.20 E-05 & 0.0564\end{array}$

0.0269

0.133

1.7896

$0.4697 \quad 6.74 E-06 \quad 1.59 E-05 \quad 1.35 E-04 \quad 9.79 E-04 \quad 0.006501$
0.217673

0.217673

0.217673

0.217673

0.141947

0.141947

0.217673

0.056486

0.056486

0.056486

0.056486

0.056486

0.056486

0.056486

0.056486

0.036856

0.056486

0.056486

0.056486

0.056486

0.056486

0.056486

0.056486

0.02694

4.306725

Drum $\$ 9607979$

\begin{tabular}{|c|c|c|c|c|c|c|c|c|c|c|c|c|}
\hline & & & Sr90 & Y90 & Tc99 & Cs137 & Eu154 & Np237 & Pu238 & Pu239 & Pu241 & \\
\hline & & 10.8 & 2.7 & $1.00 \mathrm{E}+10$ & 24. & 13.5 & 13.5 & E-03 & & IE-03 & 0.27 & \\
\hline & $.07 E-04$ & 5.19E-07 & 3.29E-05 & $8.87 E-15$ & 5.17E-07 & $1.67 \mathrm{E}-05$ & $2.23 E-06$ & 7.95E-08 & $1.96 \mathrm{E}-06$ & 1.21E-05 & $2.32 E-07$ & 7.39E-04 \\
\hline
\end{tabular}

- Bottle\#

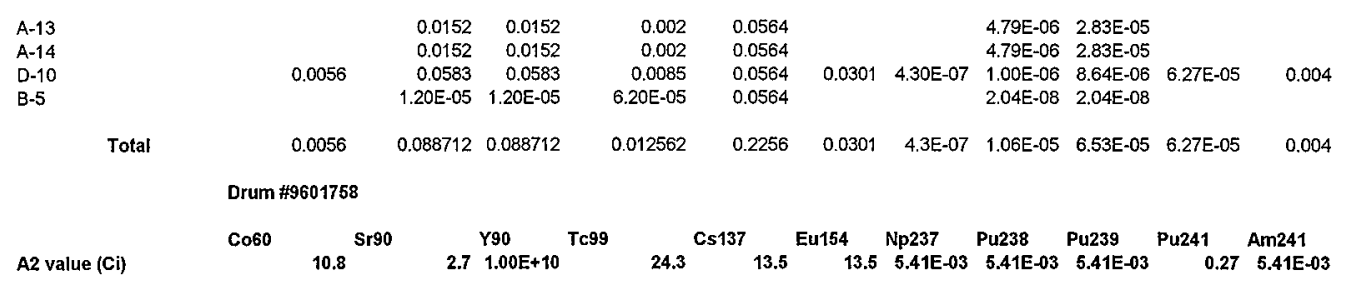

Total

0.088833

0.088833

0.221273

0.056486

0.455425 
Prepared By:

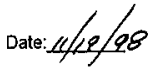

\# of A2s

8.88E-02

$6.19 \mathrm{E}-05$

$2.56 \mathrm{E}-03 \quad 6.92 \mathrm{E}-13$

4.13E-05 5.03E-04 2.64E-04 4.60E-05 2.06E-04 $\quad 1.83 \mathrm{E}-03 \quad 2.67 \mathrm{E}-04 \quad 8.30 \mathrm{E}-02$

Bottle\#

BNFL-Vit144
BNFL-Vit145
BNFL-Vit148
BNFL-Vit149
BNFL-Vit150
BNFL-Vit151
BNFL-Vit152
BNFL-Vit147

rotal

$\begin{array}{rrr}0.028 & 0.307 & 0.307 \\ 0.0509 & 0.5254 & 0.5254 \\ 0.2585 & 2.6702 & 2.6702 \\ 0.0641 & 0.6623 & 0.6623 \\ 0.0641 & 0.6623 & 0.6623 \\ 0.0385 & 0.3974 & 0.3974 \\ 0.0641 & 0.6623 & 0.6623 \\ 0.1 & 1.0332 & 1.0332 \\ 0.6682 & 6.9201 & 6.9201\end{array}$

\subsection{5}

0.0763

0.3877

0.0962

0.0962

0.0577

0.0962

0.15

1.0048

\subsection{5}

0.5086

2.5848

0.6411

0.6411

0.6411

0.6411
1.0001

6.7965 $\begin{array}{lllll}0.15 & 0.0002 & 1.00 \mathrm{E}-03 & 8.90 \mathrm{E}-03 & 6.50 \mathrm{E}-02\end{array}$ $0.2711 \quad 3.90 \mathrm{E}-06 \quad 9.02 \mathrm{E}-06 \quad 7.79 \mathrm{E}-05 \quad 5.66 \mathrm{E}-04$ $1.3778 \quad 2.00$ E-05 $\quad$ 4.59E-05 3.96E-04 2.87E-03 0.3417 4.90E-06 $1.14 \mathrm{E}-05$ 9.82E-05 7.19E-04 $0.3417 \quad 4.90 \mathrm{E}-06 \quad 1.14 \mathrm{E}-05 \quad 9.82 \mathrm{E}-05 \quad 7.19 \mathrm{E}-04$

$0.205 \quad 2.90 \mathrm{E}-06 \quad 6.82 \mathrm{E}-06 \quad 5.89 \mathrm{E}-05 \quad 4.28 \mathrm{E}-04$

$\begin{array}{lllll}0.3417 & 4.90 \mathrm{E}-06 & 1.14 \mathrm{E}-05 & 9.82 \mathrm{E}-05 & 7.19 \mathrm{E}-04\end{array}$

$0.5331 \quad 7.60 \mathrm{E}-06 \quad 1.77 \mathrm{E}-05 \quad 1.53 \mathrm{E}-04 \quad 1.11 \mathrm{E}-03$

$3.5621 \quad 0.000249 \quad 1.11 \mathrm{E}-03 \quad 9.88 \mathrm{E}-03 \quad 7.21 \mathrm{E}-02$
0.405

0.0035

0.0179

0.0044

0.0044

0.0027

0.0044

0.0069

0.4492
Total

1.7116

.96185

9.970436

2.472934

2.472934

1.483896

2.472934

3.857791

26.40438

\section{Drum \#9601835}

\begin{tabular}{|c|c|c|c|c|c|c|c|c|c|c|c|c|}
\hline & & 60 & Sr9o & Y90 & Tc99 & Cs137 & Eu154 & Np237 & Pu238 & Pu239 & Pu241 & Am \\
\hline & & 10.8 & 2.7 & $1.00 E+10$ & 24.3 & 13.5 & $\quad 13.5$ & $5.41 E-03$ & 5.41E-03 & 5.41E-03 & 0.27 & \\
\hline & 1.21 E-01 & $0.00 E+00$ & $3.19 E-02$ & $8.62 E-12$ & 4.58E-04 & 2.37E-02 & $0.00 E+00$ & $0.00 E+00$ & $5.03 E-03$ & 2.97E-02 & $.00 E+00$ & 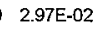 \\
\hline
\end{tabular}

Bottle\#
BNF-A-Alk-2
BNF-A-Filtrate-1
BNF-A-Filtrate-3
BNF-A-1-Alk
BNF-A-2-Alk-2
BNF-A-3-Alk
BNF-A-Prod
BNF-A-5-Alk
BNF-A-4-Alk-1

Total

$\begin{array}{rrrr}4.2366 & 4.2366 & 0.547 & 15.7451 \\ 33.8931 & 33.8931 & 4.3757 & 125.9608 \\ 33.8931 & 33.8931 & 4.3757 & 125.9608 \\ 6.8817 & 6.8817 & 0.8885 & 25.5751 \\ 2.1206 & 2.1206 & 0.2738 & 7.8811 \\ 0.5336 & 0.5336 & 0.0689 & 1.9831 \\ 0.121 & 0.121 & 0.0156 & 0.4496 \\ 0.2638 & 0.2638 & 0.0341 & 0.9804 \\ 4.2366 & 4.2366 & 0.547 & 15.7451 \\ & & & \\ 86.1801 & 86.1801 & 11.1263 & 320.2811\end{array}$

Total

24.78244

198.2598

198.2598

40.25481

12.40473

3.121364

0.707664

.543175

24.78244

504.1163

\section{Drum \#9701835}

\begin{tabular}{|c|c|c|c|c|c|c|c|c|c|c|c|c|}
\hline & & & Sr90 & Y90 & Tc99 & Cs137 & Eu154 & Np237 & Pu238 & Pu239 & Pu241 & Am241 \\
\hline & & 10.8 & 2.7 & $1.00 \mathrm{E}+10$ & 24.3 & 13.5 & 13.5 & 5.41E-03 & $5.41 \mathrm{E}-03$ & 5.41E-03 & 0.27 & \\
\hline & $1.02 E+00$ & $0.00 \mathrm{E}+00$ & $2.81 \mathrm{E}-02$ & $7.59 \mathrm{E}-12$ & $0.00 \mathrm{E}+00$ & $1.96 \mathrm{E}-02$ & $0.00 E+00$ & $0.00 E+00$ & $2.55 \mathrm{E}-02$ & $1.03 E-01$ & $0.00 E+00$ & 8.4 \\
\hline
\end{tabular}

Bottle\# 
Prepared By:Lety Date:1/19/88

$13174-070$
$15053-023$

15285-038

Tota

\begin{tabular}{ll}
12.654 & 12.654 \\
12.654 & 12.654 \\
12.654 & 12.654 \\
\hline & \\
\hline
\end{tabular}

$75.924 \quad 75.924$
Checked By:A. E.GL' Date: $11 \cdot 19.98$

$\begin{array}{rllll}44.174 & 0.023 & 0.0933 & 0.7592 & 70.3575 \\ 44.174 & 0.023 & 0.0933 & 0.7592 & 70.3575 \\ 44.174 & 0.023 & 0.0933 & 0.7592 & 70.3575 \\ & & & & \\ 265.044 & 0.138 & 0.5598 & 4.5552 & 422.145\end{array}$

\section{Drum \#9701844}

\begin{tabular}{|c|c|c|c|c|c|c|c|c|c|c|c|c|}
\hline & & & Sr90 & Y90 & Tc99 & Cs137 & Eu154 & Np237 & Pu238 & Pu239 & Pu241 & \\
\hline & & 10.8 & 2.7 & $1.00 \mathrm{E}+10$ & 24.3 & 13.5 & 13.5 & $5.4 \mathrm{tE}-03$ & & $41 E-03$ & 0.27 & \\
\hline & $1.19 E+00$ & $0.00 E+00$ & $3.41 E-02$ & $9.11 \mathrm{E}-12$ & $0.00 E+00$ & $2.38 \mathrm{E}-02$ & $.0 .00 E+00$ & $0.00 E+00$ & 3.10E-02 & 1.50E-01 & $0.00 E+00$ & $9.51 E-01$ \\
\hline
\end{tabular}

Bottle\#

BNFL-Vit-112

BNF-C-13-ELU-1

BNF-C-13-ELU-2

BNF C-2-NEU-1

BNF-CP-1-ELU

BNF-CP-2-ELU

BNF-C-9-NEU

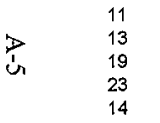

BNF-C-5-ELU

Total

$\begin{array}{rrr}15.8175 & 15.8175 & 55.2175 \\ 23.7263 & 23.7263 & 82.8236 \\ 23.7263 & 22.7263 & 82.8236 \\ 9.1742 & 9.1742 & 32.0262 \\ 6.327 & 6.327 & 22.087 \\ 7.9088 & 7.9088 & 27.6088 \\ 3.1637 & 3.1637 & 11.044 \\ 0.2253 & 0.2253 & 0.7864 \\ 0.2253 & 0.2253 & 0.7864 \\ 0.0354 & 0.0354 & 0.1236 \\ 0.0734 & 0.0734 & 0.2562 \\ 0.0987 & 0.0987 & 0.3446 \\ 1.5818 & 1.5818 & 5.5218\end{array}$

$92.0837 \quad 91.0837$
321.4497
$2.88 \mathrm{E}-02 \quad 0.0933$

4.30E-02 1.40E-01

4.30E-02 1.40E-01

$1.70 \mathrm{E}-02 \quad 5.40 \mathrm{E}-02$

1.20E-02 3.70E-02

0.014 4.70E-02

$0.0058 \quad 0.1582$

4.14E-04 1.13E-02

4.14E-04 1.13E-01

$6.52 \mathrm{E}-05 \quad 1.77 \mathrm{E}-03$

1.35E-04 3.67E-03

1.82E-04 4.94E-03

$3.00 \mathrm{E}-03 \quad 9.00 \mathrm{E}-03$

$$
\begin{array}{r}
0.7592 \\
1.138 \\
1.138 \\
0.4403 \\
0.3037 \\
3.80 \mathrm{E}-01 \\
0.1898 \\
0.0135 \\
0.0135 \\
0.0021 \\
0.0044 \\
0.0059 \\
0.759
\end{array}
$$

1.68E-01 0.813135
Total

87.7338

131.5972

130.5972

50.8859

\begin{tabular}{|c|c|c|c|c|c|c|c|c|c|c|c|c|}
\hline & & & Sr90 & Y90 & Tc99 & $\operatorname{Cs} 137$ & Eu154 & Np237 & Pu238 & Pu239 & Pu241 & \\
\hline & & 10.8 & 2.7 & $1.00 E+10$ & 24.3 & 13.5 & 13 & $.41 \mathrm{E}-03$ & $5.41 E-03$ & 41E- 03 & 0.27 & \\
\hline & $1.27 E+00$ & $0.00 E+00$ & $2.72 \mathrm{E}-02$ & $7.35 \mathrm{E}-12$ & $0.00 E+00$ & $1.90 \mathrm{E}-02$ & $0.00 E+00$ & $0.00 \mathrm{E}+00$ & $2.47 \mathrm{E}-02$ & 4.36E-01 & $0.00 E+00$ & 7.67E-01 \\
\hline
\end{tabular}

35.0937

43.867

17.7252

1.262174

1.363914

0.19833

0.411204

0.553017

9.4564

5.147

\section{Drum \#9608111}

Bottle\#

BNF-C-6-Alk
BNF-C-7-Alk
BNF-C-8-NEU
BNF-C-P-3-ALK
BNF-CP-4-ALK
BNF-C-2-NEU-2
BNF-C-3-ALK
BNF-C-4-ALK

$\begin{array}{rrr}31.637 & 31.637 & 110.44 \\ 4.7456 & 4.7456 & 16.566 \\ 4.7456 & 4.7456 & 16.566 \\ 6.327 & 6.327 & 22.087 \\ 6.327 & 6.327 & 22.087 \\ 7.9088 & 7.9088 & 27.6088 \\ 0.2253 & 0.2253 & 0.7864 \\ 7.9088 & 7.9088 & 27.6088\end{array}$

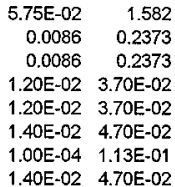

1.898

0.2847

0.2847

0.3037

0.3037

0.3796

0.135

0.3796
Total

177.2515

26.5878

26.5878

35.0937

35.0937

43.867

1.4851

43.867 
Prepared By:lat/4a/ Date: $4 / 4 / 98$

BNF-C-11-ALK
BNF-C-12-ALK

Total

\begin{tabular}{rr}
3.1635 & 3.1635 \\
0.5536 & 0.5536 \\
\hline 73.5422 & 73.5422 \\
\hline
\end{tabular}

Checked By: \& \&.Cahe Date: 11.19+98

11.04
1.9326
256.7226

$6.00 \mathrm{E}-03 \quad 1.90 \mathrm{E}-02$

0.1518

0.0266

17.5438

3.0704

$\begin{array}{lll}1.34 \mathrm{E}-01 \quad 2.3596 & 4.1474\end{array}$

410.4478

Drum \#9607625 (8 Gallon)

\begin{tabular}{|c|c|c|c|c|c|c|c|c|c|c|c|c|}
\hline & & & Sr90 & Y90 & Tc99 & Cs137 & Eu154 & Np237 & Pu238 & Pu239 & Pu241 & Am241 \\
\hline A2 value (Ci) & & 10.8 & 2.7 & $1.00 E+10$ & 24.3 & 13.5 & 13.5 & $5.41 \mathrm{E}-03$ & $5,41 E-03$ & 5.41E-03 & 0.27 & $5.41 \mathrm{E}-03$ \\
\hline \# of A2s & $6.01 E-01$ & $0.00 E+00$ & 1.14E-02 & $3.09 \mathrm{E}-12$ & $0.00 E+00$ & $7.99 E-03$ & $0.00 \mathrm{E}+00$ & $0.00 E+00$ & $1.04 \mathrm{E}-02$ & 2.41E-01 & $0.00 E+00$ & $3.30 E-01$ \\
\hline
\end{tabular}

Total

Bottie\#

BNF-C-1-ALK
BNF-C-10-ALK
ENV-C-H-3
ENV-C-H-2

Total

$\begin{array}{rrr}2.3726 & 2.3726 & 8.2826 \\ 3.1635 & 3.1635 & 11.04 \\ 12.683 & 12.683 & 44.274 \\ 12.683 & 12.683 & 44.274 \\ & & \\ 30.9021 & 30.9021 & 107.8706\end{array}$

4.00E-03 1.40E-02

6.00E-03 1.90E-02

2.31E-02 0.6341

$2,31 \mathrm{E}-02 \quad 0.634 t$

5.62E-02 1.30E $\div 00$

0.1518

0.761

0.761

1.7877

13.1597

17.5438

71.0582

71.0582

Drum \#9608011

o.

\# of A2s

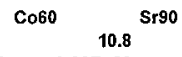

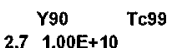

24.3

Cs137 Eu154 Np237 Pu238 Pu239 Pu241 Am241

$\begin{array}{rrrrrrrrrr}2.40 E-05 & 6.48 E-15 & 0.00 E+00 & 1.65 E-05 & 0.00 E+00 & 0.00 E+00 & 2.17 E-05 & 5.91 E-04 & 0.00 E+00 & 6.65 E-04\end{array}$

Bottle\#

$\begin{array}{lll}0.0018 & 0.0018 & 0.0062 \\ 0.0018 & 0.0018 & 0.0062 \\ 0.0018 & 0.0018 & 0.0062 \\ 0.0018 & 0.0018 & 0.0062 \\ 0.0018 & 0.0018 & 0.0062 \\ 0.0018 & 0.0018 & 0.0062 \\ 0.0018 & 0.0018 & 0.0062 \\ 0.0018 & 0.0018 & 0.0062 \\ 0.0018 & 0.0018 & 0.0062 \\ 0.0018 & 0.0018 & 0.0062 \\ 0.0018 & 0.0018 & 0.0062 \\ 0.0018 & 0.0018 & 0.0062 \\ 0.0018 & 0.0018 & 0.0062 \\ 0.0018 & 0.0018 & 0.0062 \\ 0.0018 & 0.0018 & 0.0062 \\ 0.0018 & 0.0018 & 0.0062 \\ 0.0018 & 0.0018 & 0.0062\end{array}$

Total

0.009992

0.009992

0.009992

0.009992

0.009992

0.009992

0.009992

0.009992

0.009992

0.009992

0.009992

0.009992

0.009992

0.009992

0.009992

0.009992

0.009992 
Prepared By:L/Ag/ Date:-1//9/98

$0.0018 \quad 0.0018$

$0.0018 \quad 0.0018$

$0.0018 \quad 0.0018$

$0.0018 \quad 0.0018$

$0.0018 \quad 0.0018$

$0.0018 \quad 0.0018$

$0.0018 \quad 0.0018$

$0.0018 \quad 0.0018$

$0.0018 \quad 0.0018$

$0.0018 \quad 0.0018$

$0.0018 \quad 0.0018$

$0.0018 \quad 0.0018$

$0.0018 \quad 0.0018$

$0.0018 \quad 0.0018$

$0.0018 \quad 0.0018$

$0.0018 \quad 0.0018$

$0.0648 \quad 0.0648$
$0.0018 \quad 0.0018$

$0.0018 \quad 0.0018$

$0.0018 \quad 0.0018$

Checked By: f.E.Caht Date: $11 \cdot 19 \cdot 4 Q^{\prime}$

0.0062
0.0062
0.0062
0.0062
0.0062
0.0062
0.0062
0.0062
0.0062
0.0062
0.0062
0.0062
0.0062
0.0062
0.0062
0.0062
0.0062
0.0062
0.0062

0.2232

3.27E-06 8.88E-05

0.000

0.0001

0.0001

$3.27 \mathrm{E}-06 \quad 8.88 \mathrm{E}-05$

$3.27 E-06 \quad 8.88 \mathrm{E}-05$

3.27E-06 8.88E-05

3.27E-06 8.88E-05

3.27E-06 8.88E-05

3.27E-06 $\quad 8.88 \mathrm{E}-05$

$3.27 \mathrm{E}-06 \quad 8.88 \mathrm{E}-05$

3.27E-06 8.88E-05

$3.27 \mathrm{E}-06 \quad 8.88 \mathrm{E}-05$

3.27E-06 8.88E-05

3.27E-06 8.88E-05

$3.27 \mathrm{E}-06 \quad 8.88 \mathrm{E}-05$

$3.27 \mathrm{E}-06 \quad 8.88 \mathrm{E}-05$

3.27E-06 8.88E-05

3.27E-06 8.88E-05

3.27E-06 8.88E-05

$3.27 \mathrm{E}-06 \quad 8.88 \mathrm{E}-05$

0.0001

0.0001

0.000

0.0001

0.0001

0.0001

0.0001

0.0001

0.0001

0.0001

0.0001

0.0001

0.000

0.0001

0.0001

1.18E-04 3.20E-03

0.009992

0.009992

0.009992

0.009992

0.009992

0.009992

0.009992

0.009992

0.009992

0.009992

0.009992

0.009992

0.009992

0.009992

0.009992

0.009992

0.009992

0.009992

0.009992

0.359714

Drum \#9607983

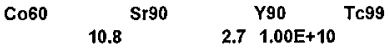

Cs137 Eu154 Np237 Pu238 Pu239 Pu241 Am241 13.5 $\begin{array}{llll}13.5 & 5.41 E-03 & 5.41 E-03 & 5.41 E-03\end{array}$ $0.27 \quad 5.41 E-03$ $A 2$ value $(\mathrm{C} i)$ $5.60 \mathrm{E}-04$ $0.00 \mathrm{E}+00$ $1.49 \mathrm{E}-04 \quad 4.02 \mathrm{E}-14$ 2.18E-06

$1.11 \mathrm{E}-04$

Bottie\#

$\begin{array}{lrrrr}\text { A-1 } & 0.0152 & 0.0152 & 0.002 & 0.0564 \\ \text { A-2 } & 0.0099 & 0.0099 & 0.0013 & 0.0368 \\ \text { A-3 } & 0.0152 & 0.0152 & 0.002 & 0.0564 \\ \text { A-4 } & 0.0099 & 0.0099 & 0.0013 & 0.0368 \\ \text { A-5 } & 0.0152 & 0.0152 & 0.002 & 0.0564 \\ \text { A-6 } & 0.0099 & 0.0099 & 0.0013 & 0.0368 \\ \text { A-7 } & 0.0152 & 0.0152 & 0.002 & 0.0564 \\ \text { A-8 } & 0.0099 & 0.0099 & 0.0013 & 0.0368 \\ \text { A-9 } & 0.0152 & 0.0152 & 0.002 & 0.0564 \\ \text { A-10 } & 0.0152 & 0.0152 & 0.002 & 0.0564 \\ \text { A-10A } & 0.0099 & 0.0099 & 0.0013 & 0.0368 \\ \text { A-10B } & 0.0099 & 0.0099 & 0.0013 & 0.0368 \\ \text { A-11 } & 0.0152 & 0.0152 & 0.002 & 0.0564 \\ \text { A-11A } & 0.0152 & 0.0152 & 0.002 & 0.0564 \\ \text { A-11B } & 0.0152 & 0.0152 & 0.002 & 0.0564\end{array}$

$\begin{array}{ll}4.79 \mathrm{E}-06 & 2.83 \mathrm{E}-05 \\ 3.12 \mathrm{E}-06 & 1.85 \mathrm{E}-05 \\ 4.79 \mathrm{E}-06 & 2.83 \mathrm{E}-05 \\ 3.12 \mathrm{E}-06 & 1.85 \mathrm{E}-05 \\ 4.79 \mathrm{E}-06 & 2.83 \mathrm{E}-05 \\ 3.12 \mathrm{E}-06 & 1.85 \mathrm{E}-05 \\ 4.79 \mathrm{E}-06 & 2.83 \mathrm{E}-05 \\ 3.12 \mathrm{E}-06 & 1.85 \mathrm{E}-05 \\ 4.79 \mathrm{E}-06 & 2.83 \mathrm{E}-05 \\ 4.79 \mathrm{E}-06 & 2.83 \mathrm{E}-05 \\ 3.12 \mathrm{E}-06 & 1.85 \mathrm{E}-05 \\ 3.12 \mathrm{E}-06 & 1.85 \mathrm{E}-05 \\ 4.79 \mathrm{E}-06 & 2.83 \mathrm{E}-05 \\ 4.79 \mathrm{E}-06 & 2.83 \mathrm{E}-05 \\ 4.79 \mathrm{E}-06 & 2.83 \mathrm{E}-05\end{array}$

$1.80 \mathrm{E}-05$

2.80E-05

$1.80 \mathrm{E}-05$

2.80E-05

$1.80 \mathrm{E}-05$

$2.80 \mathrm{E}-05$

$1.80 \mathrm{E}-05$

2.80E-05

2.80E-05

$1.80 \mathrm{E}-05$

1.80E-05

2.80E-05

2.80E-05

2.80E-05
Total

0.088861

0.05794

0.088861

0.05794

0.088861

0.05794

0.088861

0.05794

0.088861

0.088861

0.05794

0.05794

0.088861

0.088861

0.088861 

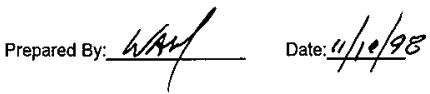

\begin{tabular}{|c|c|c|c|c|}
\hline$A-11 C$ & 0.0152 & 0.0152 & 0.002 & 0.0564 \\
\hline A-12 & 0.0152 & 0.0152 & 0.002 & 0.0564 \\
\hline A-15 & 0.0099 & 0.0099 & 0.0013 & 0.0368 \\
\hline A-16 & 0.0152 & 0.0152 & 0.002 & 0.0564 \\
\hline A-17 & 0.0099 & 0.0099 & 0.0013 & 0.0368 \\
\hline A-18 & 0.0099 & 0.0099 & 0.0013 & 0.0368 \\
\hline A-19 & 0.0152 & 0.0152 & 0.002 & 0.0564 \\
\hline$A-20$ & 0.0152 & 0.0152 & 0.002 & 0.0564 \\
\hline A-21 & 0.0152 & 0.0152 & 0.002 & 0.0564 \\
\hline A-22 & 0.0152 & 0.0152 & 0.002 & 0.0564 \\
\hline A-23 & 0.0099 & 0.0099 & 0.0013 & 0.0368 \\
\hline A- 24 & 0.0099 & 0.0099 & 0.0013 & 0.0368 \\
\hline A- 25 & 0.0152 & 0.0152 & 0.002 & 0.0564 \\
\hline A- 27 & 0.0099 & 0.0099 & 0.0013 & 0.0368 \\
\hline A- 26 & 0.0099 & 0.0099 & 0.0013 & 0.0368 \\
\hline A-28 & 0.0152 & 0.0152 & 0.002 & 0.0564 \\
\hline Total & 0.4023 & 0.4023 & 0,0529 & 1.4936 \\
\hline
\end{tabular}

$479 E 06 \quad 283 E 05$

4.79E-06 2.83E-05

3.12E-06 1.85E-05

4.79E-06 2.83E-05

3.12E-06 1.85E-05

3.12E-06 1.85E-05

4.79E-06 2.83E-05

4.79E-06 2.83E-05

4.79E-06 2.83E-05

4.79E-06 2.83E-05

3.12E-06 1.85E-05

3.12E-06 1.85E-05

4.79E-06 2.83E-05

$3.12 E-06 \quad 1.85 E-05$

3.12E-06 1.85E-05

4.79E-06 2.83E-05

1.27E-04 7.50E-04
2.80E-05

2.80E-05

$1.80 \mathrm{E}-05$

2.80E-05

1.80E-05

$1.80 \mathrm{E}-05$

280 E-05

2.80E-05

2.80E-05

$280 \mathrm{E}-05$

1.80E-05

$1.80 \mathrm{E}-05$

$2.80 \mathrm{E}-05$

$1.80 \mathrm{E}-05$

$1.80 \mathrm{E}-05$

2.80E-05

7.38E-04
0.088861

0.088861

0.05794

0.088861

0.05794

0.05794

0.088861

0.088861

0.088861

0.088861

0.05794

0.05794

0.088861

0.05794

0.05794

0.088861

2.352714

Drum \#9608010

\& A2 value (Ci)

\# of A2s $\quad 0.000256397$ Bottle\#

\begin{tabular}{lll}
25 & 0.0018 & 0.0018 \\
26 & 0.0018 & 0.0018 \\
41 & 0.0018 & 0.0018 \\
30 & 0.0018 & 0.0018 \\
40 & 0.0018 & 0.0018 \\
28 & 0.0018 & 0.0018 \\
16 & 0.0018 & 0.0018 \\
& & \\
\multicolumn{1}{r}{ Total } & 0.0126 & 0.0126
\end{tabular}

Drum $\$ 9802764$

A2 value (Ci)

Co60

$10.8^{\mathrm{Sr} 90}$

Sr90

\# of A2s 0.000682829

$\begin{array}{lll}0.7 & 0.000 \mathrm{E}+10\end{array}$

Bottle\#

6

BNFL-VIT-146
$0.0162 \quad 0.0564$

$0.0162 \quad 0.0564$
Tc99 24.3

\begin{tabular}{rrrrrrrrr}
\multicolumn{2}{c}{ Cs137 } & \multicolumn{2}{c}{ Eu154 } & Np237 & Pu238 & Pu239 & Pu241 & Am241 \\
& 13.5 & 13.5 & $\mathbf{5 . 4 1 E}-03$ & $\mathbf{5 . 4 1 E - 0 3}$ & $\mathbf{5 . 4 1 E - 0 3}$ & $\mathbf{0 . 2 7}$ & $\mathbf{5 . 4 1 E - 0 3}$ \\
0 & $3.215 \mathrm{E}-06$ & 0 & $0.00 \mathrm{E}+00$ & $4.23 \mathrm{E}-06$ & $1.15 \mathrm{E}-04$ & 0 & $1.29 \mathrm{E}-04$
\end{tabular}

0.0062
0.0062
0.0062
0.0062
0.0062
0.0062
0.0062
0.0434

3.27E-06 8.88E-05

0.0001

3.27E-06 8.88E-05 0.0001

$\begin{array}{lll}3.27 \mathrm{E}-06 & 8.88 \mathrm{E}-05 & 0.0001\end{array}$

3.27E-06 8.88E-05 0.0001

$327 \mathrm{E}-06 \quad 8.88 \mathrm{E}-05 \quad 0.0001$

3.27E-06 8.88E-05 0.0001

3.27E-06 $\quad 8.88 \mathrm{E}-05 \quad 0.0001$

$2.29 \mathrm{E}-05 \quad 6.22 \mathrm{E}-04$

0.0007

Total

0.009992

0.009992

0.009992

0.009992

0.009992

0.009992

0.009992

0.069944 
Prepared By:L/4y Date $1 / 19 / 98$

Total

$$
0.0324 \quad 0.1128
$$

\section{Drum \#9802658}

A2 value (Ci)
$\begin{aligned} & \text { \# of A2s } 0.002048487 \\ & \text { Bottle\# }\end{aligned}$

1
2
3
4
5
7

Total

$\begin{array}{llll}\mathrm{C} 060 & \mathrm{Sr} 90 & \mathrm{Y} 90 & \\ & 10.8 & 2.7 & 1.00 \mathrm{E}+10\end{array}$ Tc99

$0.000036 \quad 3.38 \mathrm{E}-14$

\begin{tabular}{|c|c|c|c|}
\hline \multicolumn{2}{|c|}{ Y90 } & Tc99 & Cs137 \\
\hline 2.7 & $1.00 \mathrm{E}+10$ & 24.3 & 13.5 \\
\hline .000036 & $3.38 \mathrm{E}-14$ & 0 & 0.0000072 \\
\hline 0.0162 & 0.0564 & & 0.0162 \\
\hline 0.0162 & 0.0564 & & 0.0162 \\
\hline 0.0162 & 0.0564 & & 0.0162 \\
\hline 0.0162 & 0.0564 & & 0.0162 \\
\hline 0.0162 & 0.0564 & & 0.0162 \\
\hline 0.0162 & 0.0564 & & 0.0162 \\
\hline 0.0972 & 0.3384 & & 0.0972 \\
\hline
\end{tabular}

Drum \#9703517

Total

A2 value (Ci)
\# of A2s 0.00957555
Bottle-t
BNF-B-1-ALK
BNF-B-FILT-4
BNF-B-FILT-3
BNF-B-FILT-2
BNF-B-FILT-5
BNF-B-2-ALK
BNF-B-FILT-1
BNFL-B-3
BNF-B-3-ALK
BNFL-Filtrate6
BNFL-B-2
BNFL-B-4
BNFL-B-1

Total

\begin{tabular}{|c|c|c|c|c|c|}
\hline Co60 & & Sr90 & Y90 & Tc99 & Cs137 \\
\hline & 10.8 & 2.7 & $1.00 \mathrm{E}+10$ & 24.3 & 13.5 \\
\hline & 0 & $1.00707 \mathrm{E}-05$ & 2.72E-15 & 5.78584E-06 & 0.0095144 \\
\hline & & & & 0.0001 & 0.0564 \\
\hline & & 0.0001 & 0.0001 & 0.0004 & 0.4103 \\
\hline & & 0.0001 & 0.0001 & 0.0004 & 0.4103 \\
\hline & & 0.0001 & 0.0001 & 0.0004 & 0.4103 \\
\hline & & 0.0001 & 0.0001 & 0.0006 & 0.5086 \\
\hline & & & & 0.0001 & 0.1154 \\
\hline & & 0.0001 & 0.0001 & 0.0004 & 0.4103 \\
\hline & & 0.002504 & 0.002504 & 0.012947 & 11.8131 \\
\hline & & & & 0.0002 & 0.2137 \\
\hline & & 0.016675 & 0.016675 & 0.086208 & 78.6571 \\
\hline & & 0.002504 & 0.002504 & 0.012947 & 11.8131 \\
\hline & & 0.002504 & 0.002504 & 0.012947 & 11.8131 \\
\hline & & 0.002504 & 0.002504 & 0.012947 & 11.8131 \\
\hline & & 0.027191 & 0.027191 & 0.140596 & 128.4448 \\
\hline
\end{tabular}

Tol $\begin{array}{llll}0.027191 & 0.027191 & 0.140596 & 128.4448\end{array}$

$\begin{array}{ll}2.04 \mathrm{E}-08 & 2.04 \mathrm{E}-08 \\ 1.49 \mathrm{E}-07 & 1.48 \mathrm{E}-07 \\ 1.49 \mathrm{E}-07 & 1.48 \mathrm{E}-07 \\ 1.49 \mathrm{E}-07 & 1.49 \mathrm{E}-07 \\ 1.84 \mathrm{E}-07 & 1.84 \mathrm{E}-07 \\ 4.18 \mathrm{E}-08 & 4.18 \mathrm{E}-08 \\ 1.49 \mathrm{E}-07 & 1.49 \mathrm{E}-07 \\ 4.28 \mathrm{E}-06 & 4.28 \mathrm{E}-06 \\ 7.74 \mathrm{E}-08 & 7.74 \mathrm{E}-08 \\ 2.85 \mathrm{E}-05 & 2.85 \mathrm{E}-05 \\ 4.28 \mathrm{E}-06 & 4.28 \mathrm{E}-06 \\ 4.28 \mathrm{E}-06 & 4.28 \mathrm{E}-06 \\ 4.28 \mathrm{E}-06 & 4.28 \mathrm{E}-06 \\ & \\ 4.65 \mathrm{E}-05 & 4.65 \mathrm{E}-05\end{array}$

Pu241 Am241 $\begin{array}{llllll}5.5 & 5.41 E-03 & 5.41 E-03 & 5.41 E-03 & 0.27 & 5.41 E-03\end{array}$

$\begin{array}{rlllll}0 & 0.00 E+00 & 3.26 E-15 & 8.96 E-04 & 0 & 1.11 E-03\end{array}$

$$
\begin{array}{ll}
2.94 \mathrm{E}-15 & 8.08 \mathrm{E}-04 \\
2.94 \mathrm{E}-15 & 8.08 \mathrm{E}-04 \\
2.94 \mathrm{E}-15 & 8.08 \mathrm{E}-04 \\
2.94 \mathrm{E}-15 & 8.08 \mathrm{E}-04 \\
2.94 \mathrm{E}-15 & 8.08 \mathrm{E}-04 \\
2.94 \mathrm{E}-15 & 8.08 \mathrm{E}-04 \\
1.76 \mathrm{E}-14 & 4.85 \mathrm{E}-03
\end{array}
$$$$
0.001
$$$$
0.001
$$$$
0.001
$$$$
0.001
$$$$
0.001
$$$$
0.001
$$$$
0.006
$$

Total

Total
0.090608

0.090608

0.090608

0.090608

0.090608

0.090608

0.543649

0.1155

0.4109

1.42E-05

11.83108

0.2139

9.50E-05

1.42E-05

78.77681

11.83108

11.83108

1.42E-05

11.83108

0.000152 
Prepared By:

\section{Drum $\$ 9703473$}

\begin{tabular}{llllllllllllll} 
Co60 & Sr90 & \multicolumn{1}{c}{ Y90 } & Tc99 & \multicolumn{2}{c}{ Cs137 } & Eu154 & Np237 & Pu238 & Pu239 & Pu241 Am241 \\
& 10.8 & & 2.7 & $1.00 E+10$ & & 24.3 & 13.5 & 13.5 & $5.41 E-03$ & $5.41 E-03$ & $5.41 E-03$ & 0.27 & $5.41 E-03$
\end{tabular}

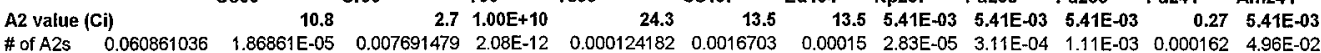

Bottle\#

\section{BNF-B-ELU-3}

BNFL-Vit-153

BNFL-B-ELU-2

BNFL-Vit-154

BNF-B-ELU-1

BNFL-Vit-163A

BNFL-Vit-155A

BNFL-VIt-156A

Total

$\begin{array}{rrr} & 0.0002 & 0.0002 \\ 0.1 & 1.0332 & 1.0332 \\ & 0.0001 & 0.0001 \\ 0.1 & 1.0332 & 1.0332 \\ & 0.0001 & 0.0001 \\ 0.001771 & 18.29659 & 18.29659 \\ & 4.95 \mathrm{E}-05 & 4.95 \mathrm{E}-05 \\ 3.91 \mathrm{E}-05 & 0.403554 & 0.403554\end{array}$

$0.2018101 \quad 20.7669935 \quad 20.76699$

$\begin{array}{rrrrrrrrr}0.0007 & 1.0001 & & & 3.62 \mathrm{E}-07 & 3.62 \mathrm{E}-07 & & \\ 0.15 & 1.0001 & 0.5331 & 7.60 \mathrm{E}-06 & 1.77 \mathrm{E}-04 & 1.31 \mathrm{E}-04 & 1.11 \mathrm{E}-03 & 0.0069 \\ 0.0007 & 0.6069 & & & 2.20 \mathrm{E}-07 & 2.20 \mathrm{E}-07 & & \\ 0.15 & 1.0001 & 0.5331 & 7.60 \mathrm{E}-06 & 1.77 \mathrm{E}-04 & 1.31 \mathrm{E}-04 & 1.11 \mathrm{E}-03 & 0.0069 \\ 0.0007 & 0.6069 & & & 2.20 \mathrm{E}-07 & 2.20 \mathrm{E}-07 & & \\ 2.656665 & 17.7111 & 0.944104 & 0.000135 & 0.001298 & 0.005606 & 0.040692 & 0.253957 \\ 0.000256 & 0.23336 & & & 8.45 \mathrm{E}-08 & 8.45 \mathrm{E}-08 & & 2.82 \mathrm{E}-07 \\ 0.058596 & 0.39064 & 0.020823 & 2.99 \mathrm{E}-06 & 2.86 \mathrm{E}-05 & 0.000124 & 0.000898 & 0.00056 \\ & & & & & & & \\ 3.017617 & 22.5492 & 2.031127 & 0.000153 & 1.68 \mathrm{E}-03 & 5.99 \mathrm{E}-03 & 0.043814 & 0.268317\end{array}$

Total

1.001201

3.857928

0.6078

3.857928

0.6078

58.20851

0.233715

$1.28 \mathrm{E}+00$

69.6537 
HNF-3622, Rev. 0

APPENDIX B

\section{RADCALC FILE}




\section{HNF-3622, Rev. 0}

Date: $\quad 11-19-98 \quad 09: 27$

Performed By: W.A.M Loemick

checked By: $\frac{4 . \varepsilon . \text { Cols }}{\pi} \| / 19 / 98$

$$
\begin{aligned}
& \text { Isotope Data output File: SAV_WATT.DAT }
\end{aligned}
$$

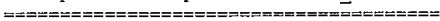

\begin{tabular}{|c|c|c|}
\hline ISOTOPE & $\begin{array}{c}\text { ACTIVITY } \\
(\mathrm{Bq})\end{array}$ & $\begin{array}{l}\text { ACTIVITY } \\
\text { (Ci) }\end{array}$ \\
\hline $5 r-90$ & $3.40 \mathrm{E}+009$ & $9.20 \mathrm{E}-002$ \\
\hline$+Y-90$ & $3.37 \mathrm{E}+009$ & $9.10 \mathrm{E}-002$ \\
\hline $\operatorname{cs}-137$ & $1.19 \mathrm{E}+010$ & $3.21 \mathrm{E}-001$ \\
\hline$+\mathrm{Ba}-137 \mathrm{~m}$ & 1. $12 \mathrm{E}+010$ & $3.04 \mathrm{E}-001$ \\
\hline$* * \mathrm{pu}-238$ & $6.22 E+006$ & $1.68 \mathrm{E}-004$ \\
\hline$\star \star \mathrm{Pu}-239$ & $3.00 \mathrm{E}+007$ & $8.10 \mathrm{E}-00 \mathrm{~A}$ \\
\hline${ }^{*} \mathrm{Am}-241$ & $1.89 \mathrm{E}+008$ & $5.10 \mathrm{E}-003$ \\
\hline TOTAL & $3.01 \mathrm{E}+0$ & $8.14 \mathrm{E}-001$ \\
\hline & $1.55 \mathrm{E}+010$ & $4.19 \mathrm{E}-001$ \\
\hline
\end{tabular}

\begin{tabular}{|c|c|c|c|}
\hline ISOTOPE & $\begin{array}{c}\text { Number } \\
\text { of } \\
\text { A2s }\end{array}$ & $\begin{array}{c}\text { Eraction } \\
\text { of Total } \\
\text { A2s }\end{array}$ & $\begin{array}{c}\text { Cumulative } \\
\text { Total } \\
\text { A2s }\end{array}$ \\
\hline 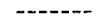 & ----ー-ー- & - & \\
\hline$A m-241$ & $9.43 \mathrm{E}-001$ & $7.98 \mathrm{E}-001$ & 0.798000 \\
\hline $\mathrm{Qu}-239$ & $1.50 \mathrm{E}-001$ & $1.272-001$ & 0.924741 \\
\hline$s r-90$ & $3.41 \mathrm{E}-002$ & $2.88 E-002$ & 0.953585 \\
\hline $\mathrm{Pu}-238$ & $3.11 \mathrm{E}-002$ & $2.63 E-002$ & 0.979872 \\
\hline Cs -137 & $2.38 \mathrm{E}-002$ & $2.01 E-002$ & 1.00000 \\
\hline & $0 \quad 00 \mathrm{E}+000$ & $0.00 \mathrm{E}+000$ & 1.00000 \\
\hline & & & 1.00000 \\
\hline
\end{tabular}$$
\text { Input Eile: SAV WATT.RAD - Initial source }
$$

Shipping papers and labels:

\begin{tabular}{c} 
SPECIEIC \\
ACTIVITY \\
$(\mathrm{Ci} / \mathrm{g})$ \\
\hline $1.41 \mathrm{E}+002$ \\
$5.43 \mathrm{E}+005$ \\
$8.70 \mathrm{E}+001$ \\
$5.38 \mathrm{E}+008$ \\
$1.70 \mathrm{E}+001$ \\
$6.20 \mathrm{E}-002$ \\
$3.43 \mathrm{E}+000$ \\
\\
$($ excluding \\
\\
\\
\\
\\
\\
\\
Total \\
A2s \\
\hline 0.798000 \\
0.924741 \\
0.953585 \\
1.979872 \\
1.000000 \\
1.00000
\end{tabular}

* Contains $95 \%$ of the total number of A2s and must be included per 49 CFR 173.433 . 


\begin{tabular}{|c|c|c|c|c|c|}
\hline \multicolumn{6}{|c|}{ DISTRIBUTION SHEET } \\
\hline \multirow{2}{*}{$\begin{array}{l}\text { To } \\
\text { Distribution }\end{array}$} & \multirow{2}{*}{\multicolumn{3}{|c|}{$\begin{array}{l}\text { From } \\
\text { Packaging Engineering }\end{array}$}} & \multicolumn{2}{|l|}{ Page 1 of 1} \\
\hline & & & & \multicolumn{2}{|c|}{ Date Nov. 19, 1998} \\
\hline \multicolumn{4}{|l|}{ Project Title/Work Order } & \multicolumn{2}{|c|}{ EDT No. 622971} \\
\hline \multicolumn{4}{|c|}{$\begin{array}{l}\text { Safety Evaluation for Packaging (Onsite) Savannah River Sample } \\
\text { Drums (HNF-3622, Rev. 0) }\end{array}$} & ECN No. NA & \\
\hline Name & MSIN & $\begin{array}{l}\text { Text } \\
\text { With All } \\
\text { Attach. }\end{array}$ & Text Only & $\begin{array}{l}\text { Attach./ } \\
\text { Appendix } \\
\text { Only }\end{array}$ & $\begin{array}{l}\text { EDT/ECN } \\
\text { Only }\end{array}$ \\
\hline $\begin{array}{l}\text { R. L. Clawson } \\
\text { J. G. Field } \\
\text { T. A. Frazier } \\
\text { W. A. McCormick } \\
\text { J. E. Mercado } \\
\text { T. Romano } \\
\text { R. J. Smith } \\
\text { M. R. Turner } \\
\text { J. L. Westcott }\end{array}$ & $\begin{array}{l}H 1-14 \\
H 1-15 \\
T 6-20 \\
H 1-15 \\
H 1-15 \\
H 1-15 \\
H 1-15 \\
H 1-15 \\
T 3-04\end{array}$ & $\begin{array}{l}X \\
X \\
X \\
X \\
X \\
X \\
X \\
X \\
X\end{array}$ & & & \\
\hline $\begin{array}{l}\text { HNF-2622 File } \\
\text { RL Reading Room } \\
\text { Work Control (D. Kelly) }\end{array}$ & $\begin{array}{l}\mathrm{H} 1-15 \\
\mathrm{H} 2-53 \\
\mathrm{H} 1-15\end{array}$ & $\begin{array}{l}x \\
x\end{array}$ & & & $x$ \\
\hline Central Files & $\mathrm{Bl}-07$ & $x$ & & & \\
\hline
\end{tabular}

